\title{
Time-Varying Biased Proportional Guidance with Seeker's Field-of-View Limit
}

\author{
Zhe Yang, Hui Wang, and Defu Lin \\ Beijing Institute of Technology, Beijing 100081, China \\ Correspondence should be addressed to Hui Wang; wh20031131@126.com
}

Received 12 November 2015; Accepted 7 February 2016

Academic Editor: Christian Circi

Copyright (C) 2016 Zhe Yang et al. This is an open access article distributed under the Creative Commons Attribution License, which permits unrestricted use, distribution, and reproduction in any medium, provided the original work is properly cited.

Traditional guidance laws with range-to-go information or time-to-go estimation may not be implemented in passive homing missiles since passive seekers cannot measure relative range directly. A time-varying biased proportional guidance law, which only uses line-of-sight (LOS) rate and look angle information, is proposed to satisfy both impact angle constraint and seeker's field-ofview (FOV) limit. In the proposed guidance law, two time-varying bias terms are applied to divide the trajectory into initial phase and terminal phase. The initial bias is designed as a function of LOS rate and look angle to maintain the seeker's lock-on while the final bias eliminates the deviation between the integral value of angle control bias and the expected bias amount. A switching logic is adopted to change the biases continuously so that there is no abrupt acceleration change during the engagement. Extensive simulations considering both kinematic and realistic missile models are performed to illustrate the efficiency of the proposed method.

\section{Introduction}

In guidance law designs, not only achieving acceptable miss distance values, but also considering impact angles has become an increasingly important necessity. In some applications, it is desirable to control the path angle of missiles such as antitank missiles and antiship missiles to a proper impact angle to enhance warhead effectiveness and lethality [1]. On the other hand, maintaining missile seeker's lock-on during the engagement is critical for successful interception, especially for narrower FOV seekers such as strapdown seeker $[2,3]$.

Guidance laws with terminal angle control have been widely studied in the literatures. Most of the advanced guidance laws used optimal control theory [4-6] or sliding mode control [7-9] to intercept the targets with angular constraint. Usually, these guidance laws include relative range- or timeto-go information which cannot be measured by passive missiles directly. Several variants of pure proportional navigation (PPN) guidance law [10] were also used for deriving impact angle control due to their effectiveness and simplicity of implementation. A biased proportional navigation guidance law was proposed in [11] by adding a time-varying term to control the flight path angle. In [12], the guidance law with
LOS information sought convergence to a circular path by using the PPN guidance law with a specific navigation gain. A two-phased proportional navigation guidance law was introduced in [13] for achieving all impact angles against stationary targets. Another two-phased structure was presented in [14], where the PPN was enhanced with a bias term during the first phase. The bias would be removed after the switching condition was achieved for the second phase.

Furthermore, seeker's FOV constraint was also considered in angle control biased guidance laws. A bias-shaping method was introduced in [15] to cope with the terminal angle constraint as well as look angle limit. In [16], the switched-gain proportional navigation guidance (SGPNG) law used $N=1$ in the first stage and shaped the missile trajectory to achieve the desired terminal angle with any $N \geq$ 2 in the second stage. A closed-form solution for the choice of navigation gains was proposed to achieve large impact angles without violating the FOV limit. Based on the same switching logic, the switched-bias guidance method in [17] used seeker's FOV maximum value to calculate the bias in the initial phase.

This paper proposes a new guidance law called timevarying biased proportional navigation (TVBPN) that uses two time-varying biases for achieving both terminal angle constraint and look angle limitation. The first-stage bias, 


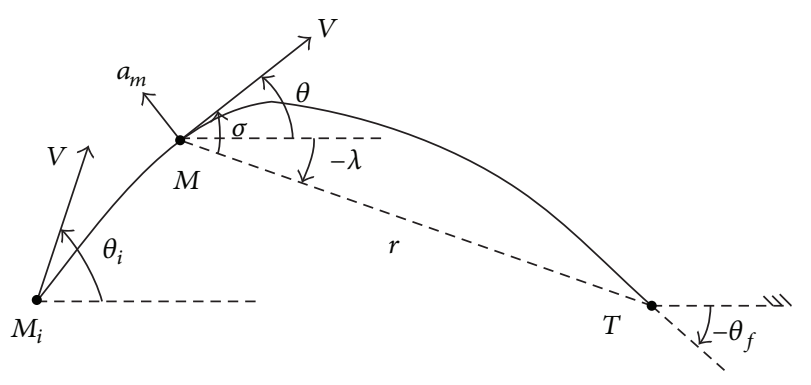

Figure 1: Engagement geometry.

which is a function of LOS rate and look angle, guides the missile to flight with the maximum look angle. The secondstage bias calculates the derivation between the integral value of bias and the expected bias amount, aiming to decrease the impact angle bias to zero before the interception. The study also derives the missile-target relative range at the switching instant and the maximum lateral acceleration analytically. The bias is no longer a certain constant but an adaptive time-varying value. The switching logic ensures that the bias changes continuously so that there is no jump in the acceleration command during the engagement. Compared with a similar guidance law in [16], simulations on a kinematic and a realistic missile model are presented.

The outline of this paper is organized as follows. The impact angle control problem is described in Section 2. Section 3 presents the details of the proposed two-stage guidance law and its switching logic, followed by the performance analysis in Section 4. Nonlinear simulation results with some comparisons are shown in Section 5 while conclusions are offered in Section 6.

\section{Problem Statement}

Consider a two-dimensional surface-to-surface engagement scenario against a stationary target in Figure 1. The initial positions of the missile and the target are denoted by $\left(x_{i}, 0\right)$ and $\left(x_{T}, 0\right), a_{m}$ is the missile lateral acceleration, which is perpendicular to the constant missile velocity $V . r, \theta, \lambda$, and $\sigma$ denote range-to-go information, flight path angle, LOS angle, and look angle, respectively. Angles are positive in the counterclockwise direction. The subscripts $i$ and $f$ denote the initial and terminal time, respectively. The relationship among the three pursuit angles is

$$
\theta=\lambda+\sigma .
$$

The seeker's FOV is defined as the angle between missile body axis and LOS angle. Considering the notion that missile's attack of angle is usually small, look angle $\sigma$ can be approximately considered as FOV angle.

The nonlinear differential equations describing the homing engagement problem can be written as

$$
\begin{aligned}
& \dot{r}=-V \cos \sigma, \\
& \dot{\lambda}=-\frac{V \sin \sigma}{r},
\end{aligned}
$$

where the dot operator represents the derivative with respect to time. The traditional PPN guidance law has the following form:

$$
a_{m}=V \dot{\theta}=N V \dot{\lambda},
$$

where $N$ is the navigation gain, $\dot{\theta}$ is flight path angular rate, and $\dot{\lambda}$ is LOS rate. Integrating (4) from $t_{i}$ to $t_{f}$, we obtain

$$
\theta_{f}=\theta_{i}+N\left(\lambda_{f}-\lambda_{i}\right) .
$$

For a stationary target, the terminal flight path angle should be equal to the LOS vector when the missile is on the collision course; that is,

$$
\theta_{f}=\lambda_{f}
$$

In addition, for surface-to-surface engagement, the initial LOS angle should be zero. Using these conditions, (5) can be rewritten as

$$
\theta_{f}=\frac{\theta_{i}}{1-N} .
$$

According to (7), the terminal impact angle with PPN is determined by the navigation gain $N$ and the initial flight path angle $\theta_{i}$. When PPN is only applied to intercept the stationary target, the achievable impact angles are $\left[\begin{array}{ll}-\theta_{i} & 0\end{array}\right]$ for $N \geq 2$. The achievable impact angle becomes smaller with larger navigation gain. Based on PPN, a biased proportional navigation (BPN) guidance law is designed in [14]. The flight path rate is obedient to the following:

$$
\dot{\theta}=N \dot{\lambda}+b,
$$

where $b$ is the bias value. The acceleration command is

$$
a_{m}=N V \dot{\lambda}+V b .
$$

Integrating (8) with initial conditions leads to

$$
\theta(t)-\theta_{i}=N\left(\lambda(t)-\lambda_{i}\right)+\int_{0}^{t} b d t .
$$

When $t=t_{f}$,

$$
\theta_{f}=\theta_{i}+N\left(\lambda_{f}-\lambda_{i}\right)+\int_{0}^{t_{f}} b d t .
$$

BPN impacts the terminal angle by adjusting the integral of b. Substituting (6) into (11) yields

$$
\int_{0}^{t_{f}} b d t=(1-N) \theta_{f}-\theta_{i} .
$$

In (12), the expected bias amount is

$$
B_{\text {ref }}=(1-N) \theta_{f}-\theta_{i} .
$$

The integral value of bias during the engagement is

$$
B=\int_{0}^{t} b d t .
$$


In [14], BPN assumes that the bias is a constant. So (14) can be rewritten as

$$
B=b \cdot \Delta t
$$

where $\Delta t$ is the amount of time when the bias is on. In a certain time range, $\mathrm{BPN}$ is used to shape the missile trajectory by making $B$ reach $B_{\text {ref }}$ before the interception. Thereafter, the guidance law switches from BPN to PPN. The bias value is chosen as

$$
b=\frac{B_{\text {ref }}}{\Delta t}=\frac{(1-N) \theta_{f}-\theta_{i}}{\Delta t} .
$$

However, the bias should be appropriately chosen because large value will lead to high acceleration command. Considering missile acceleration limit, $\Delta t$ should be chosen as large as possible. For example, the amount of time is designed as [14]

$$
\Delta t=\frac{r_{i}}{V_{i}}
$$

where $r_{i}$ and $V_{i}$ denote the initial range and missile initial velocity, respectively.

\section{TVBPN Guidance Law Design}

In this section, the TVBPN guidance law is proposed to achieve impact angle control within seeker's FOV constraint. Different from the bias in BPN, a switching logic is adopted to choose the appropriate bias between two different timevarying biases.

The missile trajectory can be divided into two phases by two different biases. In the initial phase, the bias is proposed as

$$
b_{1}=(1-N) \dot{\lambda}+\frac{1}{\tau_{1}}\left(\sigma_{\max }-\sigma\right),
$$

where $\sigma_{\max }$ is the seeker's FOV maximum value and $\tau_{1}$ is the time constant to make $\sigma$ converge to $\sigma_{\max }$. Substituting (18) into (9), we can obtain the acceleration command in the initial phase

$$
a_{m}=V \dot{\lambda}+\frac{V}{\tau_{1}}\left(\sigma_{\max }-\sigma\right) .
$$

Proposition 1. When (19) is applied to the initial guidance phase, the look angle can asymptotically converge to the maximum value with any initial look angle.

Proof. Taking the derivative of (1) and considering (8), we get

$$
\dot{\sigma}=\dot{\theta}-\dot{\lambda}=(N-1) \dot{\lambda}+b .
$$

Substituting (18) into (20) yields

$$
\dot{\sigma}=\frac{1}{\tau_{1}}\left(\sigma_{\max }-\sigma\right) .
$$

The solution of (21) is obtained as

$$
\sigma(t)=\sigma_{\max }\left(1-\exp \left(-\frac{t}{\tau_{1}}\right)\right) .
$$

As shown in (22), $\sigma(t)$ converges to $\sigma_{\max }$ exponentially with the time constant $\tau_{1}$. Note that if $t=\tau_{1}, \sigma=0.63 \sigma_{\max }$. If $t=3 \tau_{1}, \sigma=0.95 \sigma_{\max }$. If $t=5 \tau_{1}, \sigma=0.99 \sigma_{\max }$. In other words, the look angle will asymptotically converge to the maximum value with any initial look angle. This completes the proof.

The missile is far away from the target at the initial time, so in (19) the magnitude of the LOS rate is negligible and the bias term of the right-hand side is more dominant. The maximum acceleration in the initial phase is generated at the beginning of the homing; that is,

$$
a_{1, \max }=-\frac{V^{2} \sin \sigma_{i}}{r_{i}}+\frac{V}{\tau_{1}}\left(\sigma_{\max }-\sigma_{i}\right) .
$$

The look angle will reach the maximum value quickly in the initial guidance phase if time constant $\tau_{1}$ is sufficiently small. One can imply from (23) that the acceleration command will exceed the acceleration limit if $\tau_{1}$ is too small. Therefore, $\tau_{1}$ should be determined appropriately.

As mentioned in Section 2, the purpose of the bias term is to ensure that the integral value of bias $B$ in (14) converges to $B_{\text {ref }}$ in (13). Depending on the difference between $B$ and $B_{\text {ref }}$, the second-stage bias is varied as

$$
b_{2}(t)=\dot{B}(t)=\frac{B_{\mathrm{ref}}-B(t)}{\tau_{2}},
$$

where $\tau_{2}$ is the time constant to make $B$ converge to the expected value $B_{\text {ref }}$. Solving the linear differential equation (24) yields

$$
B(t)=C \exp \left(-\frac{t}{\tau_{2}}\right)+B_{\text {ref }},
$$

where $C$ is the undetermined coefficient. Note that the integral value of bias at the switching instant is

$$
B\left(t_{s}\right)=\int_{0}^{t_{s}} b_{1}(t) d t,
$$

where $t_{s}$ and $B\left(t_{s}\right)$ denote the switching time and the integral value of bias at the switching instant, respectively. Substituting (26) into (25) yields

$$
\begin{aligned}
B(t) & =-\left(B_{\mathrm{ref}}-B\left(t_{s}\right)\right) \exp \left(-\frac{\left(t-t_{s}\right)}{\tau_{2}}\right)+B_{\mathrm{ref}}, \\
b_{2} & =\frac{B_{\mathrm{ref}}-B(t)}{\tau_{2}} \\
& =\frac{\left(B_{\mathrm{ref}}-B\left(t_{s}\right)\right) \exp \left(-\left(t-t_{s}\right) / \tau_{2}\right)}{\tau_{2}} .
\end{aligned}
$$

During the engagement, the first-stage bias $b_{1}$ increases after the look angle reaches the maximum value, while the second-stage bias $b_{2}$ decreases to zero as impact time increases. The switching logic of the time-varying bias can be expressed as

$$
b= \begin{cases}b_{1}, & \left|b_{1}\right|<\left|b_{2}\right| \\ b_{2}, & \left|b_{1}\right| \geq\left|b_{2}\right|\end{cases}
$$




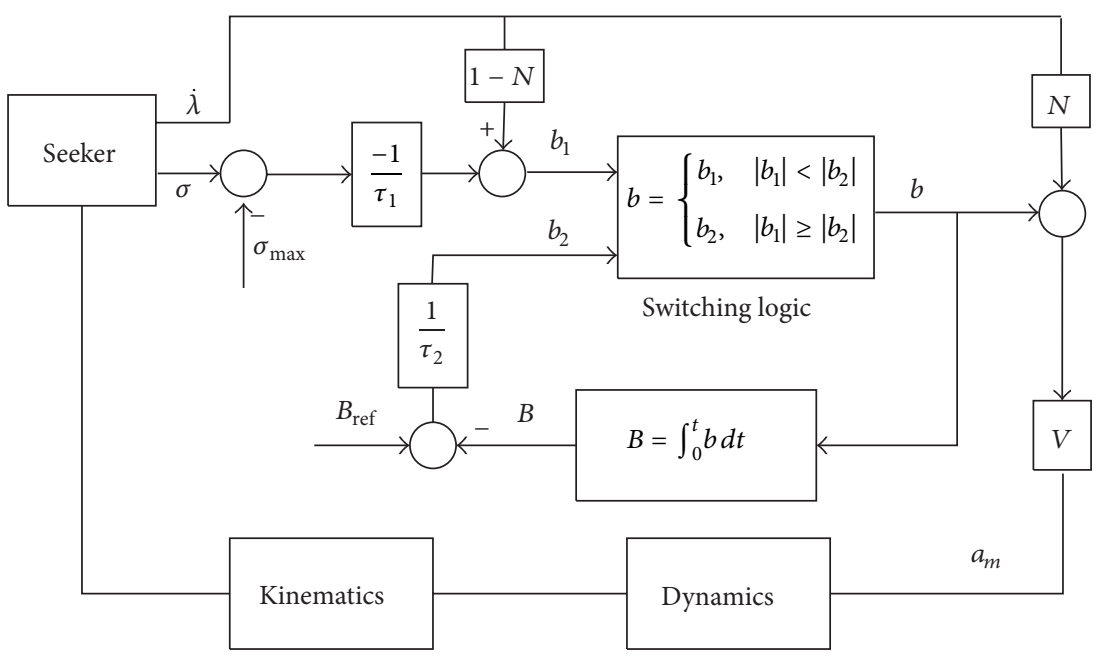

FIGURE 2: TVBPN guidance method.

Accordingly, when $\left|b_{1}\right| \geq\left|b_{2}\right|$, the bias is switched to $b_{2}$ for achieving the terminal angle with the successful interception and avoiding an abrupt acceleration change during the switching process. An abrupt command change is unfavorable for the autopilot because it may cause autopilot instability due to the actuator's slew rate limit.

The proposed TVBPN guidance method is presented in Figure 2. The scheme only requires two measurements: LOS rate and look angle, which can be obtained by passive homing seekers. Since neither range-to-go nor time-to-go information is required, the proposed guidance law seems to be more effective in practical implementation.

Proposition 2. TVBPN can maintain the seeker look angle within the FOV limit during the whole engagement.

Proof. When $\left|b_{1}\right|<\left|b_{2}\right|$, the look angle will reach the maximum value exponentially because the missile look angular rate is

$$
\dot{\sigma}(t)=(N-1) \dot{\lambda}+b_{1}=\frac{1}{\tau_{1}}\left(\sigma_{\max }-\sigma\right)
$$

The look angle rate will decrease to zero monotonously. Therefore, the look angle would not exceed the maximum value in the first guidance phase.

When $\left|b_{1}\right| \geq\left|b_{2}\right|$, substituting (28) into (20) yields

$$
\begin{aligned}
\dot{\sigma}(t)= & (N-1) \dot{\lambda}+b_{2} \\
= & \frac{(1-N) V \sin \sigma(t)}{r(t)} \\
& +\frac{\left(B_{\text {ref }}-B\left(t_{s}\right)\right) \exp \left(-\left(t-t_{s}\right) / \tau_{2}\right)}{\tau_{2}} .
\end{aligned}
$$

If $N \geq 2,0<\sigma \leq \sigma_{\max } \leq \pi / 2$, we can get two inequalities as follows:

$$
\begin{aligned}
& \frac{(1-N) V \sin \sigma(t)}{r(t)} \leq \frac{(1-N) V \sin \sigma\left(t_{s}\right)}{r\left(t_{s}\right)}<0, \\
& \frac{\left(B_{\text {ref }}-B\left(t_{s}\right)\right)}{\tau_{2}} \geq \frac{\left(B_{\text {ref }}-B\left(t_{s}\right)\right) \exp \left(-\left(t-t_{s}\right) / \tau_{2}\right)}{\tau_{2}} \\
& >0 .
\end{aligned}
$$

Adding (32) and (33) gives

$$
\begin{aligned}
\dot{\sigma}(t)= & \frac{(1-N) V \sin \sigma(t)}{r(t)} \\
& +\frac{\left(B_{\text {ref }}-B\left(t_{s}\right)\right) \exp \left(-\left(t-t_{s}\right) / \tau_{2}\right)}{\tau_{2}} \\
\leq & \frac{(1-N) V \sin \sigma\left(t_{s}\right)}{r\left(t_{s}\right)}+\frac{\left(B_{\text {ref }}-B\left(t_{s}\right)\right)}{\tau_{2}}=\dot{\sigma}\left(t_{s}\right) \\
\leq & 0 .
\end{aligned}
$$

The preceding equation shows that $\dot{\sigma}(t) \leq 0$ and $\sigma(t)<\sigma_{\max }$. As mentioned previously, the bias term is no longer needed if $B$ satisfies the expected value $B_{\text {ref }}$. Thereafter, the guidance law is changed from TVBPN to PPN. In PPN guidance law, (20) can be rewritten as

$$
\dot{\sigma}=\dot{\theta}-\dot{\lambda}=-\frac{(N-1) V \sin \sigma}{r}
$$

If (35) is divided by (2), the look angular rate with respect to relative range can be obtained as

$$
\frac{d \sin \sigma}{d r}=\frac{(N-1) \sin \sigma}{r}
$$


The solution of (36) is calculated as

$$
\frac{\sin \sigma}{\sin \sigma_{p}}=\left(\frac{r}{r_{p}}\right)^{N-1},
$$

where $\sigma_{p}$ and $r_{p}$ denote the look angle and relative range at the beginning of PPN, respectively. Equation (37) shows that the look angle decreases monotonously to zero when $N>1$.

In summary, the proposed guidance law guarantees that the FOV constraint is not violated during the whole engagement. The proposition will also be verified by the simulations in Section 5.

\section{Analysis of the Proposed Guidance Law}

4.1. The Relative Range $r_{s}$ at the Switching Instant. The firststage bias at the switching time is

$$
b_{1}\left(t_{s}\right)=\frac{(N-1) \sin \sigma_{\max }}{r_{s}} .
$$

The integral value of bias at $t=t_{s}$ can be obtained as

$$
\begin{aligned}
B\left(t_{s}\right) & =\int_{0}^{t_{s}} b_{1} d t=\int_{0}^{t_{s}}(1-N) \dot{\lambda}+\dot{\sigma} d t \\
& =\theta_{s}-N \lambda_{s}-\theta_{i} .
\end{aligned}
$$

The second-stage bias at the switching time is

$$
b_{2}\left(t_{s}\right)=\frac{B_{\mathrm{ref}}-B\left(t_{s}\right)}{\tau_{2}}=\frac{B_{\mathrm{ref}}-\left(\theta_{s}-N \lambda_{s}-\theta_{i}\right)}{\tau_{2}} .
$$

At the switching instant, we can get the relationship among the angles as follows:

$$
\theta_{s}=\lambda_{s}+\sigma_{\max }
$$

After (3) is divided by (2), the differential equation of $\lambda$ with respect to $r$ in the initial phase can be approximated as

$$
\frac{d \lambda}{d r}=\frac{\tan \sigma}{r} \approx \frac{\tan \sigma_{\max }}{r} \text {. }
$$

When $t=t_{s}$, the solution of (42) can be obtained as

$$
\lambda_{s}=\tan \sigma_{\max } \ln \frac{r_{s}}{r_{i}} .
$$

Substituting (41) and (43) into (40) gives

$$
\begin{aligned}
& b_{2}\left(t_{s}\right)=\frac{B_{\text {ref }}-B\left(t_{s}\right)}{\tau_{2}} \\
& =\frac{B_{\text {ref }}-\left((1-N) \lambda_{s}+\sigma_{\max }-\theta_{i}\right)}{\tau_{2}} \\
& =\frac{B_{\text {ref }}-\left((1-N) \tan \sigma_{\max } \ln \left(r_{s} / r_{i}\right)+\sigma_{\max }-\theta_{i}\right)}{\tau_{2}} .
\end{aligned}
$$

According to the switching logic,

$$
\left|b_{1}\left(t_{s}\right)\right|=\left|b_{2}\left(t_{s}\right)\right| \text {. }
$$

Substituting (38) and (44) into (45) yields

$$
\begin{aligned}
& \left|\frac{(N-1) V \sin \sigma_{\text {max }}}{r_{s}}\right| \\
& \quad=\left|\frac{B_{\text {ref }}-\left((1-N) \tan \sigma_{\max } \ln \left(r_{s} / r_{i}\right)+\sigma_{\max }-\theta_{i}\right)}{\tau_{2}}\right| .
\end{aligned}
$$

From (46), the relative range at the switching instant can be determined by numerical analysis methods. Then, the LOS angle $\lambda_{s}$ can be calculated by (43). Also, when $\sigma_{i}=\sigma_{\max }$, the switching time $t_{s}$ can be determined by

$$
t_{s}=\frac{r_{i}-r_{s}}{V \cos \sigma_{\max }}
$$

4.2. Lateral Acceleration Requirement. In the first guidance phase, the acceleration command decreases as the look angle converges to its maximum value exponentially. The maximum acceleration command is generated at the beginning of the engagement, which is given by

$$
a_{1, \max }=-\frac{V^{2} \sin \sigma_{i}}{r_{i}}+\frac{V}{\tau_{1}}\left(\sigma_{\max }-\sigma_{i}\right) .
$$

With $B(t)$ reaching $B_{\text {ref }}$, the guidance law is changed from TVBPN to PPN. The acceleration command in the second stage can be estimated by the acceleration of PPN:

$$
\left|a_{2}\right|=\left|N V \dot{\lambda}+V b_{2}\right| \leq|N V \dot{\lambda}|=\left|a_{\mathrm{PPN}}\right|
$$

Because the maximum acceleration of PPN occurs at the switching instant, the maximum acceleration command of TVBPN in the second stage can be approximated as

$$
a_{2, \max } \leq a_{\mathrm{PPN}, \max }=\left|N V \dot{\lambda}_{s}\right|=\frac{N V^{2} \sin \sigma_{\max }}{r_{s}} .
$$

Note that $a_{\mathrm{PPN} \text {,max }}$ is related to the range $r_{s}$, and $r_{s}$ can be determined by $\theta_{f}, \sigma_{\max }, \theta_{i}$, and $N$ in (46). Therefore, $a_{2 \text {,max }}$ can be estimated if $\theta_{f}, \sigma_{\max }, \theta_{i}$, and $N$ are known in advance.

In summary, the maximum acceleration command of TVBPN during the whole engagement can be estimated by

$$
\begin{aligned}
a_{\max } & \begin{cases}-\frac{V^{2} \sin \sigma_{i}}{r_{i}}+\frac{V}{\tau_{1}}\left(\sigma_{\max }-\sigma_{i}\right), & a_{1, \max } \geq a_{2, \max }, \\
\frac{N V^{2} \sin \sigma_{\max }}{r_{s}}, & a_{1, \max }<a_{2, \max } .\end{cases}
\end{aligned}
$$

For instance, if the engagement conditions are given as $r_{i}=5000 \mathrm{~m}, V=250 \mathrm{~m} / \mathrm{s}, \theta_{i}=\sigma_{i}=15 \mathrm{deg}, \sigma_{\max }=$ $45 \mathrm{deg}, \tau_{1}=2 \mathrm{~s}, \tau_{2}=0.5 \mathrm{~s}$, and $N=3$, the relative range $r_{s}$ at the switching instant and the maximum acceleration 
requirement $a_{\max }$ can be determined according to (46) and (51). In the case of $\theta_{f}=-90 \mathrm{deg}$, the relative range $r_{s} \approx$ $1625.3 \mathrm{~m}$, and the maximum acceleration commands in two guidance phases are $a_{1, \max }=62.21 \mathrm{~m} / \mathrm{s}^{2}$ and $a_{2, \max }=$ $81.57 \mathrm{~m} / \mathrm{s}^{2}$, respectively. Using this analytical method, the acceleration requirement of the proposed guidance law can be evaluated in advance.

\section{Simulations Results}

To demonstrate the basic performance of the proposed TVBPN, nonlinear simulations are performed with a constant speed missile model firstly. Then, a realistic missile model with given aerodynamic and thrust profiles is considered to prove the applicability of the proposed guidance law in a realistic scenario. In addition, the proposed guidance law is compared with another impact angle control guidance law which uses SGPNG.

\subsection{Constant Speed Missile Model}

Case 1 (simulations for various engagement conditions). In this case, simulations are carried out with constant missile speed model. The missile speed is $V=250 \mathrm{~m} / \mathrm{s}$. The common parameters of surface-to-surface missile engagement simulations are given as $r_{i}=5000 \mathrm{~m}, \theta_{i}=\sigma_{i}=15 \mathrm{deg}, \sigma_{\max }=$ $45 \mathrm{deg}, \tau_{1}=2 \mathrm{~s}, \tau_{2}=0.5 \mathrm{~s}$, and $N=3$. The desired impact angles are $0 \mathrm{deg},-45 \mathrm{deg},-90 \mathrm{deg},-135 \mathrm{deg}$, and $-180 \mathrm{deg}$. All simulations are terminated for $r>0.1 \mathrm{~m}$. The simulation results are represented in Figure 3. In Figure 3(a), for large impact angles $\left(\left|\theta_{f}\right| \geq\left|\theta_{i}\right|\right)$, missile trajectories are the same until the time-varying bias changes from $b_{1}$ to $b_{2}$. Figures $3(\mathrm{~b})$ and $3(\mathrm{c})$ show that all the missiles can achieve the desired impact angles from 0 deg to $-180 \mathrm{deg}$ within the FOV limit. The acceleration curves in Figure 3(d) are different from each other in the final phase because of different terminal angle constraints. One can imply that larger desired impact angle demands higher control effort. In Figure 3(e), switching instant of the time-varying biases can be clearly observed, if applicable. Biases change from initial phase to terminal phase continuously and approach zero finally. In Figure 3(f), as expected, each integral value of bias converges to its expected $B_{\text {ref }}$ at the final time. It should be pointed out that the proposed guidance law can also achieve small terminal angle constraint by choosing the second-stage bias $b_{2}$ directly (e.g., $\left.\theta_{f}=0 \mathrm{deg}\right)$.

Case 2 (comparisons with other guidance laws in a constant speed missile model). In this subsection, the guidance law in [16] is chosen to compare with the proposed guidance law. In [16], the impact angle control guidance law called SGPNG is derived considering a planar surface-to-surface engagement scenario against a stationary target. SGPNG is investigated to fly with a constant look-ahead angle with navigation gain $N_{m}=1$ before it is switched to $N=N_{s}$ for achieving impact angles. The corresponding switching condition can be derived as

$$
\Gamma=\lambda-\frac{\sigma}{N_{s}-1},
$$

where $N_{s}$ is the second-stage navigation gain.
For traditional surface-to-surface missiles, $\lambda_{i}=0$ and $\theta_{i} \geq 0$. And terminal lateral acceleration is guaranteed to be bounded only for $N_{s} \geq 2$ [18]. According to (52), the contrastive guidance law can only achieve the terminal angles which are larger than the initial flight path angle; that is, $\left|\theta_{f}\right| \geq\left|\Gamma_{i}\right|=\theta_{i}$, while the proposed guidance law does not have such constraint and can achieve a wider range of impact angles, such as the case of $\theta_{f}=0 \mathrm{deg}$ in Figure 3 .

The initial flight path angle in the proposed guidance law is $\theta_{i}=15 \mathrm{deg}$, while it is set to $\theta_{i}=\sigma_{\max }=45 \mathrm{deg}$ in SGPNG. Here, we choose $N_{m}=1$ and $N=N_{s}=3$ in the following simulations. The desired impact angles are $-90 \mathrm{deg}$ and $-120 \mathrm{deg}$. All the other simulation conditions are the same as the previous scenario in Case 1. The simulation results are shown in Figure 4. As can be seen from Figures 4(a) and 4(b), the missile trajectories using two guidance laws are similar and both can intercept the target at desired impact angles with negligible errors. In the case of $\theta_{f}=-90 \mathrm{deg}$, the impact angle errors of TVBPN and SGPNG are $0.0045 \mathrm{deg}$ and $0.0062 \mathrm{deg}$, respectively. Figure 4(c) provides the seeker look angle profiles. For TVBPN, the look angle converges exponentially to the maximum value, and then the look angle is maintained until satisfying the switching condition in (29). For SGPNG, the look angle keeps the maximum value because its first-stage navigation gain $N_{m}=1$. Then, the look angle reduces to zero when satisfying the switching condition in (52). The lateral accelerations are shown in Figure 4(d). Compared with SGPNG, TVBPN avoids the abrupt change of the acceleration command at the switching instant.

\subsection{Realistic Missile Model}

Case 3 (simulations for realistic missile model). In this subsection, further simulations are carried out with a realistic missile model considering autopilot lag. The aerodynamic and vehicle properties of the realistic missile model are taken from [19]. The equations of motion are given as

$$
\begin{aligned}
\dot{x}_{m} & =V_{m} \cos \theta, \\
\dot{y}_{m} & =V_{m} \sin \theta, \\
\dot{V}_{m} & =\frac{T-D}{m}-g \sin \theta, \\
\dot{\theta} & =\frac{a_{m}-g \cos \theta}{V_{m}} .
\end{aligned}
$$

In the case of autopilot lag, first-order uncompensated autopilot dynamics are considered with a time constant $\tau_{m}$. The autopilot dynamics lag can be expressed as

$$
a_{m}=\frac{1}{\tau_{m} s+1} a_{c},
$$

where $a_{c}$ and $a_{m}$ are the acceleration command and missile lateral acceleration, respectively.

For realistic implementation, the second-stage bias needs to be changed as

$$
b_{2}(t)=\frac{B_{\text {ref }}(t)}{\tau_{2}}=\frac{(1-N) \theta_{f}+N \lambda-\theta}{\tau_{2}},
$$




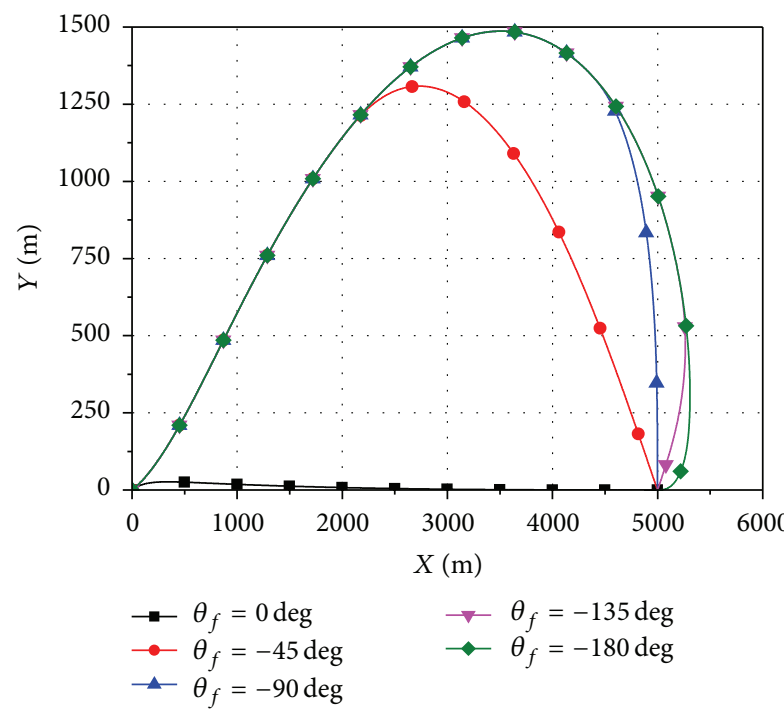

(a) Trajectory

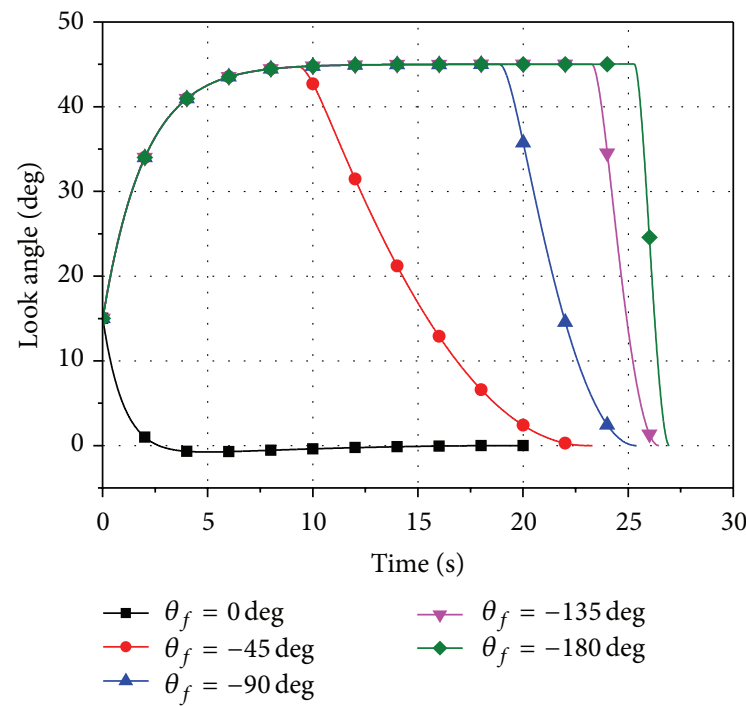

(c) Look angle

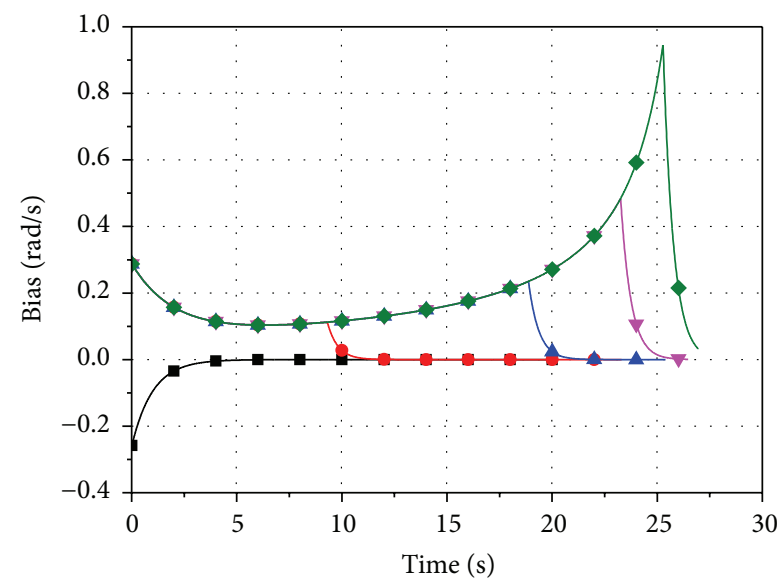

$$
\begin{aligned}
& \longrightarrow \theta_{f}=0 \mathrm{deg} \quad \longrightarrow \theta_{f}=-135 \mathrm{deg} \\
& \longrightarrow \theta_{f}=-45 \mathrm{deg} \\
& \multimap \theta_{f}=-90 \mathrm{deg}
\end{aligned}
$$

(e) Bias profiles

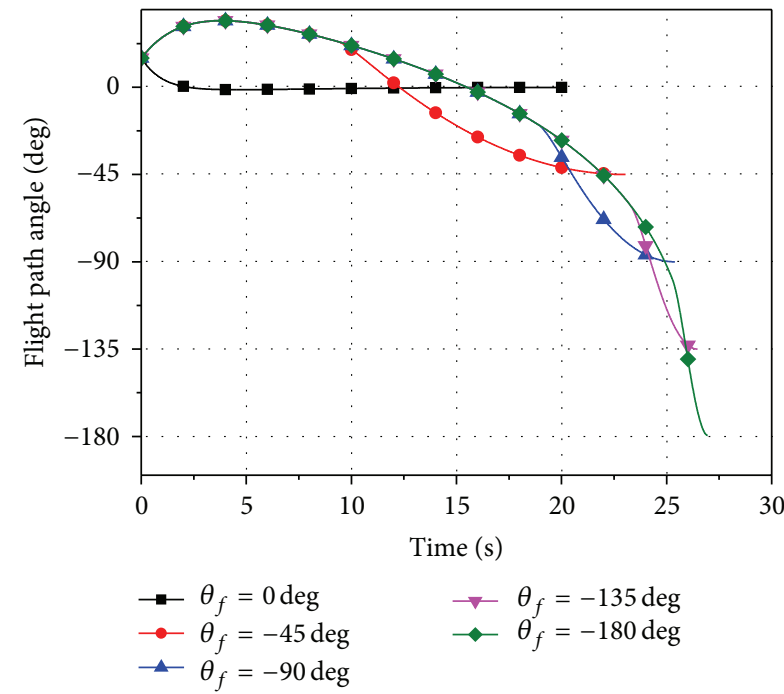

(b) Flight path angle

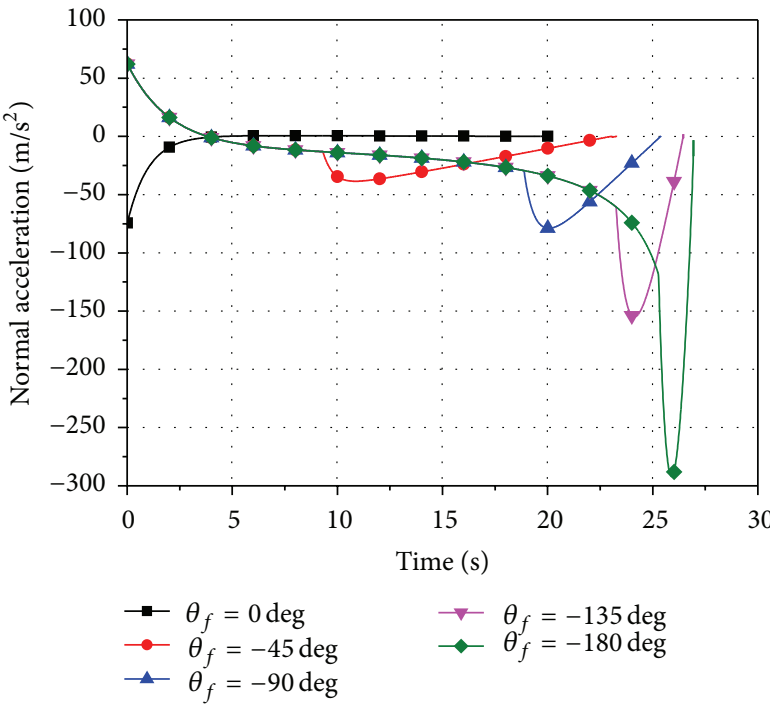

(d) Acceleration

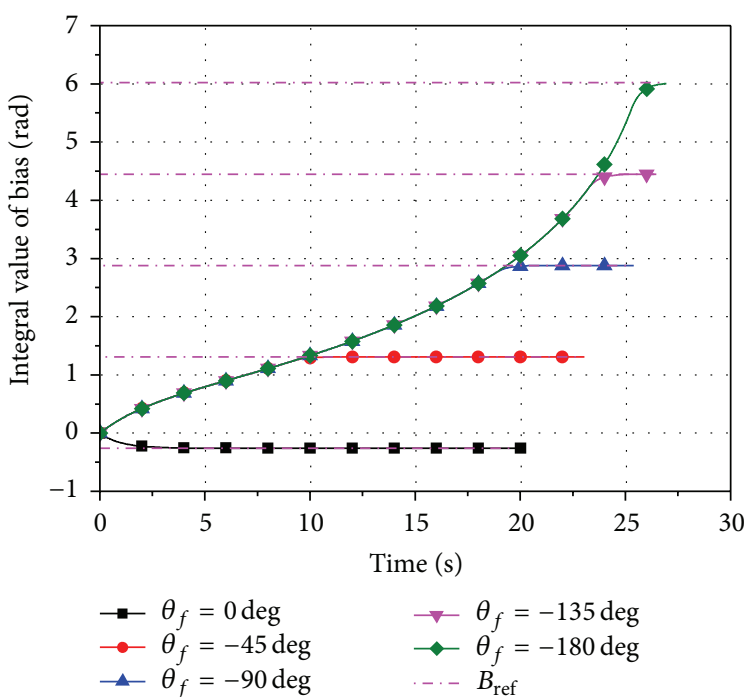

(f) Integral values of bias

FIgURE 3: Simulation results for constant speed missile model. 


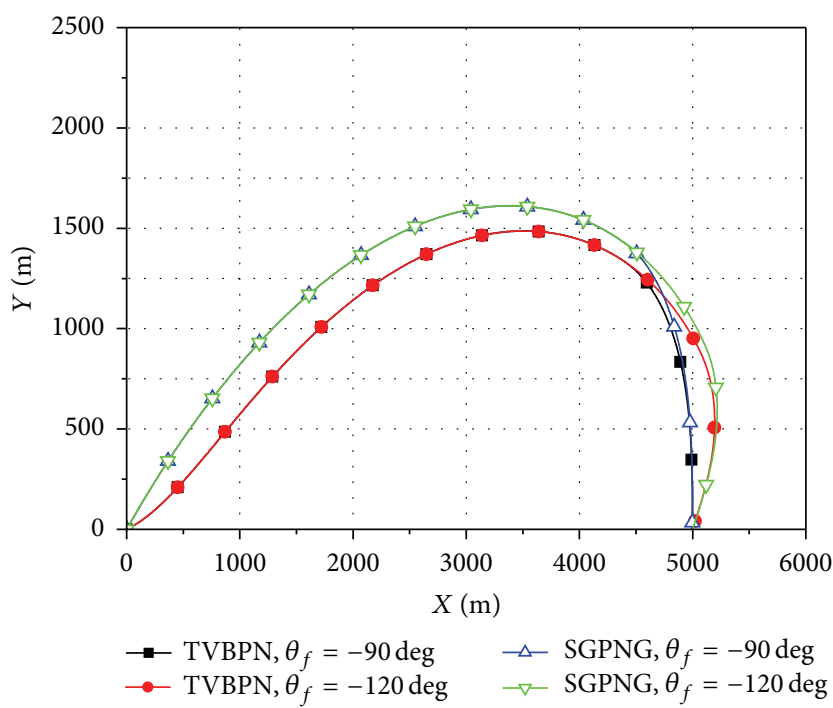

(a) Trajectory

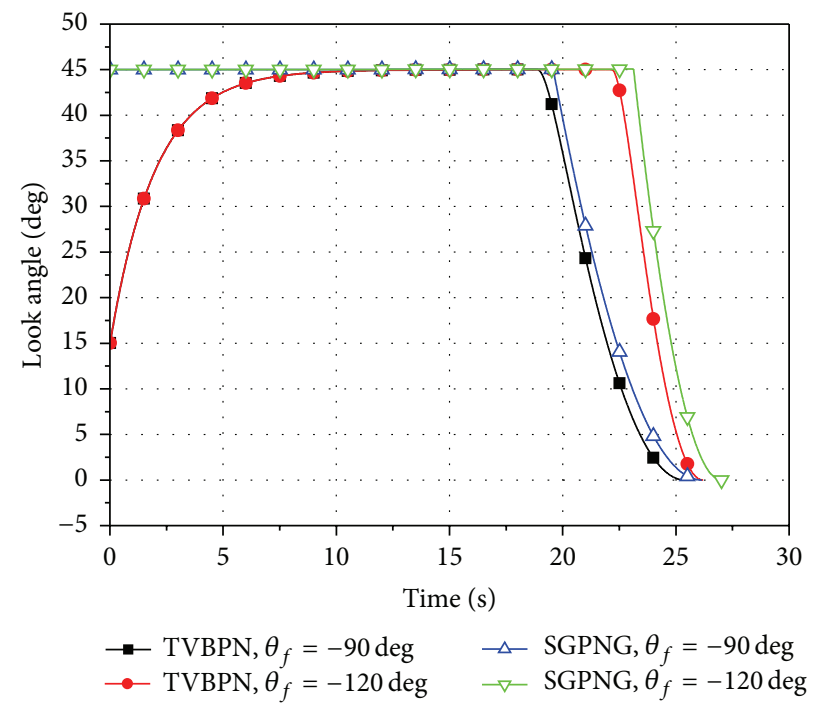

(c) Look angle

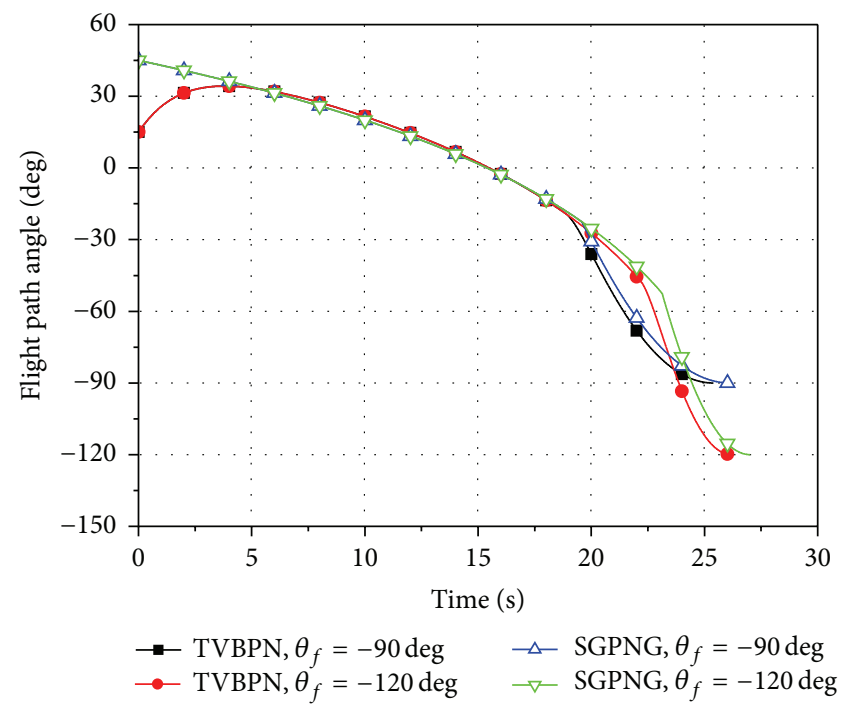

(b) Flight path angle

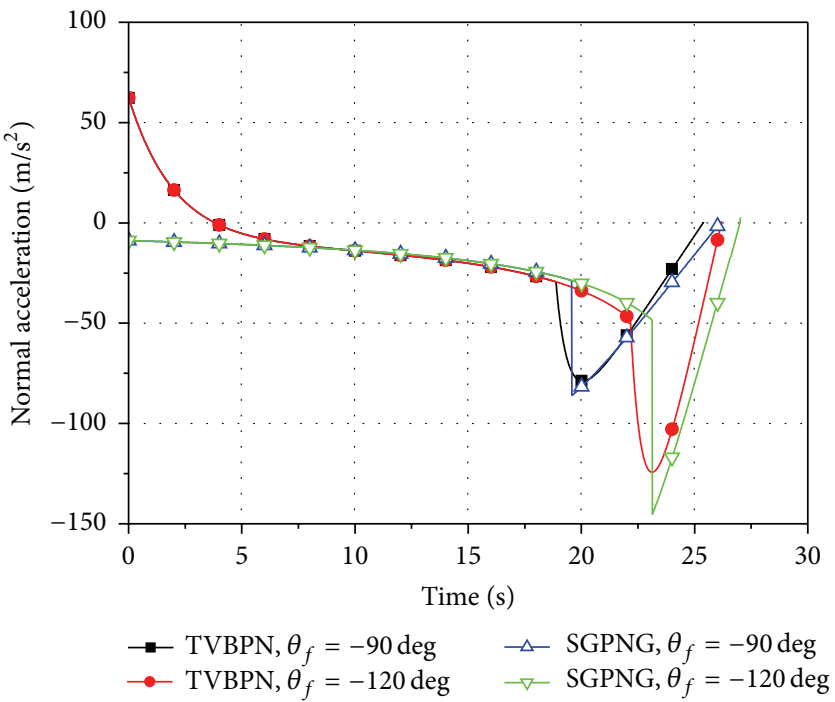

(d) Acceleration

FIGURE 4: Comparison results for constant speed missile model.

where $\theta$ and $\lambda$ are the instantaneous fight path angle and LOS angle, respectively.

The simulation conditions of this case are given by $r_{i}=$ $10000 \mathrm{~m}, \theta_{i}=\sigma_{i}=40 \mathrm{deg}, \sigma_{\max }=45 \mathrm{deg}, \tau_{1}=2 \mathrm{~s}$, $\tau_{2}=0.5 \mathrm{~s}, \tau_{m}=0.3 \mathrm{~s}$, and $N=3$. The desired impact angles are $-45 \mathrm{deg},-90 \mathrm{deg}$, and $-135 \mathrm{deg}$. Figures $5(\mathrm{a})$ and 5(b) show that the missiles successfully intercept the target with different terminal angles. As shown in Figure 5(c), the missile velocity becomes the maximum when the boost phase is finished at $t=1.5 \mathrm{~s}$, and then the speed is decreased by the gravity and the induced drag. The acceleration variation is plotted in Figure 5(d). In the simulations, the guidance loop is closed after the boost phase at $t=1.5 \mathrm{~s}$ until no lateral acceleration is applied. In Figure 5(e), the look angle in the first stage no longer corresponds to the maximum value but varies slightly in a realistic missile scenario. Figure 5(f) shows clearly that the time-varying $B_{\text {ref }}$ decreases in the early stage with TVBPN while it does not with PPN. Results highlight the satisfactory performance of the proposed guidance scheme in a realistic engagement environment.

Case 4 (comparisons with other guidance laws in a realistic missile model). In this case, the performance of the proposed guidance law is compared with SGPNG in a realistic missile model with autopilot lag. The initial flight path angle is $\theta_{i}=$ $\sigma_{i}=45 \mathrm{deg}$. With the same other conditions as in Case 3, comparison simulations are plotted in Figure 6 with desired impact angles $-90 \mathrm{deg}$ and $-120 \mathrm{deg}$. Figures $6(\mathrm{a})-6(\mathrm{~d})$ provide the trajectories, flight path angles, look angles, and lateral accelerations under TVBPN and SGPNG, respectively. The miss distances and impact angle errors are given in Table 1. Both guidance methods show the successful interception of 


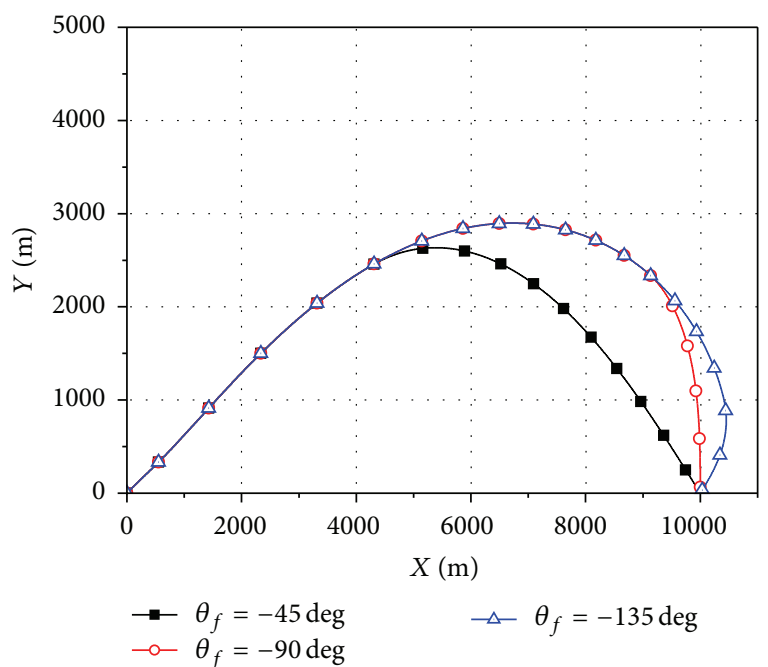

(a) Trajectory

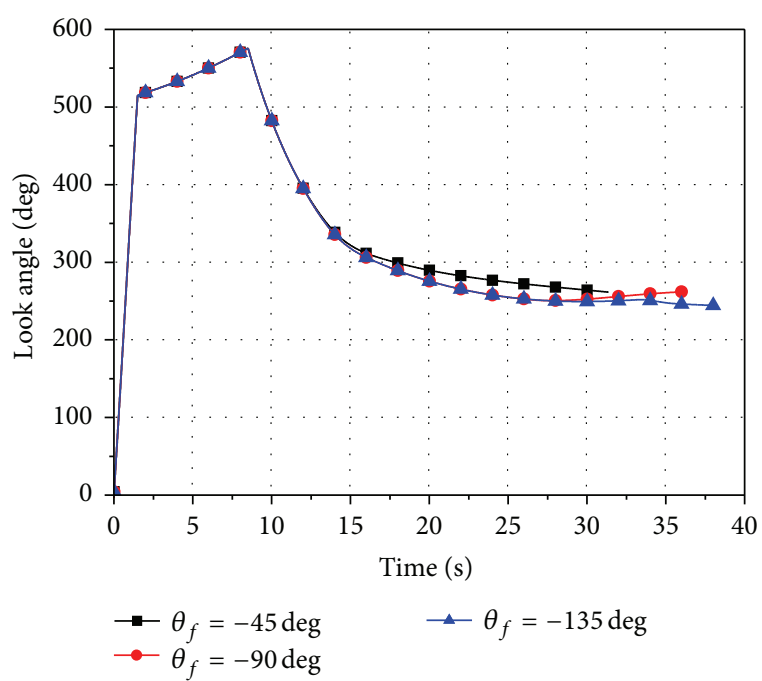

(c) Velocity

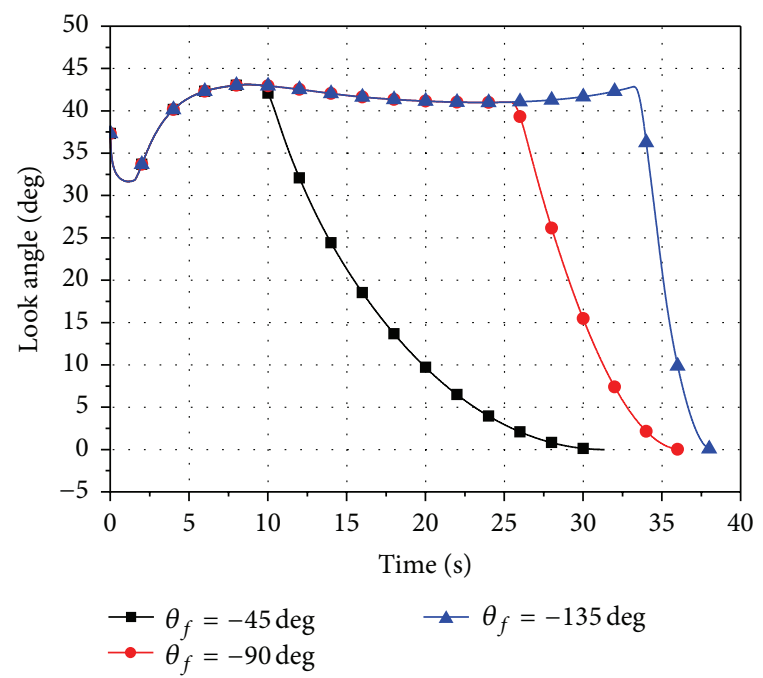

(e) Look angle

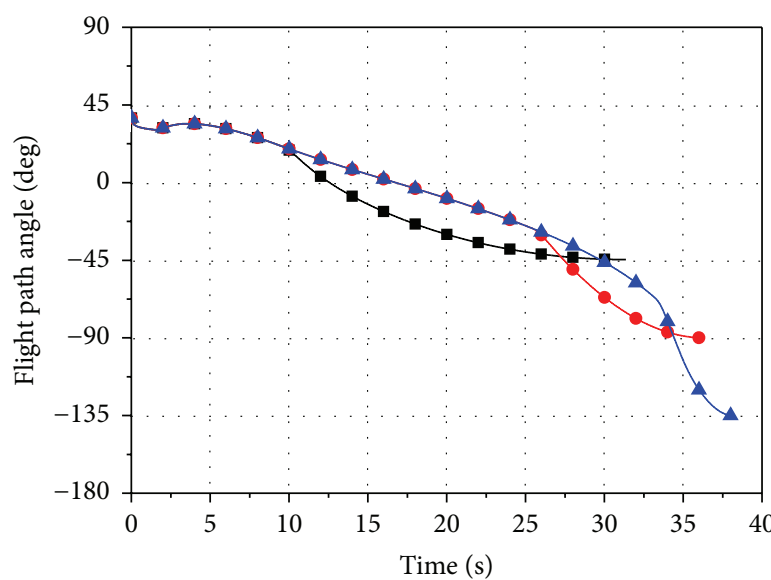

$$
\begin{aligned}
& \longrightarrow \theta_{f}=-45 \mathrm{deg} \quad \longrightarrow \theta_{f}=-135 \mathrm{deg} \\
& \multimap \theta_{f}=-90 \mathrm{deg}
\end{aligned}
$$

(b) Flight path angle

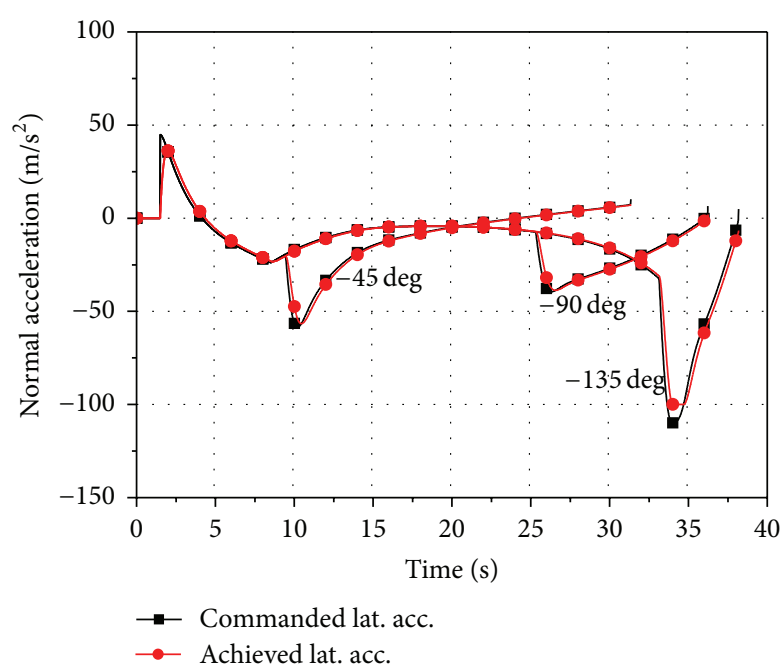

(d) Acceleration

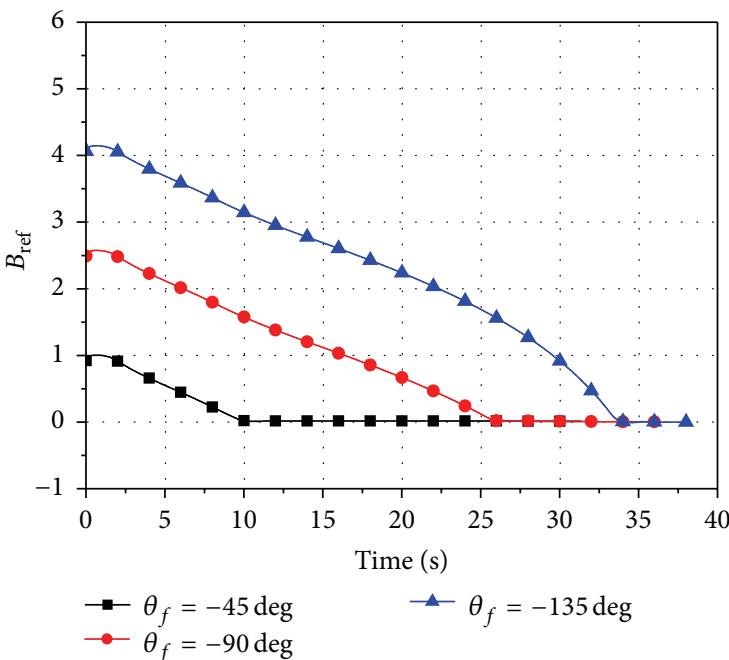

(f) Integral values of bias

FIgURE 5: Simulation results for realistic missile model. 


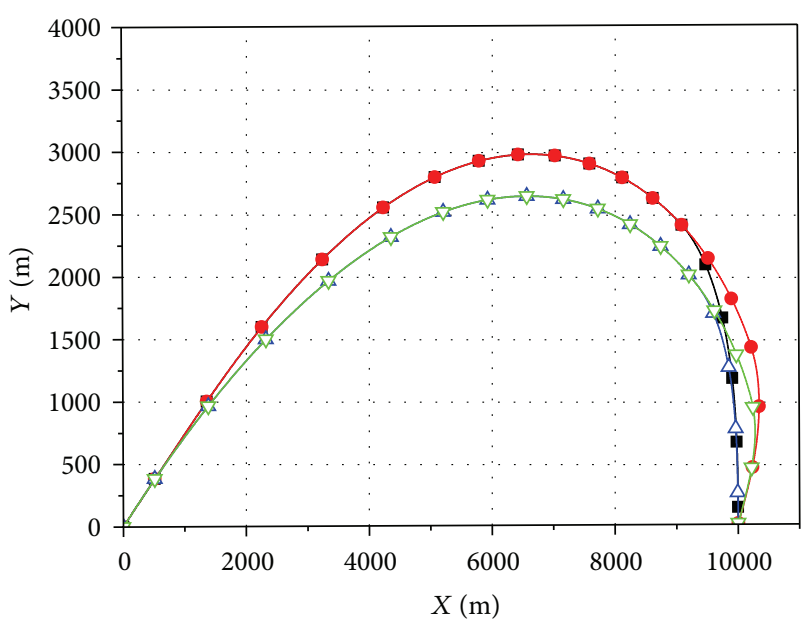

$\rightarrow$ TVBPN, $\theta_{f}=-90 \mathrm{deg} \quad \neg-$ SGPNG, $\theta_{f}=-90 \mathrm{deg}$ $\rightarrow$ TVBPN, $\theta_{f}=-120 \mathrm{deg} \quad \rightarrow-$ SGPNG, $\theta_{f}=-120 \mathrm{deg}$

(a) Trajectory

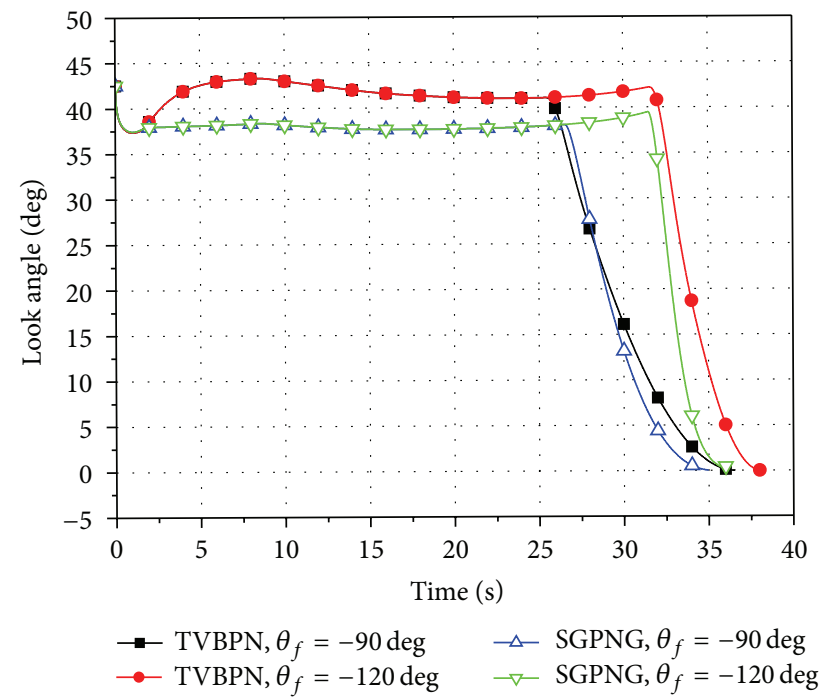

(c) Look angle

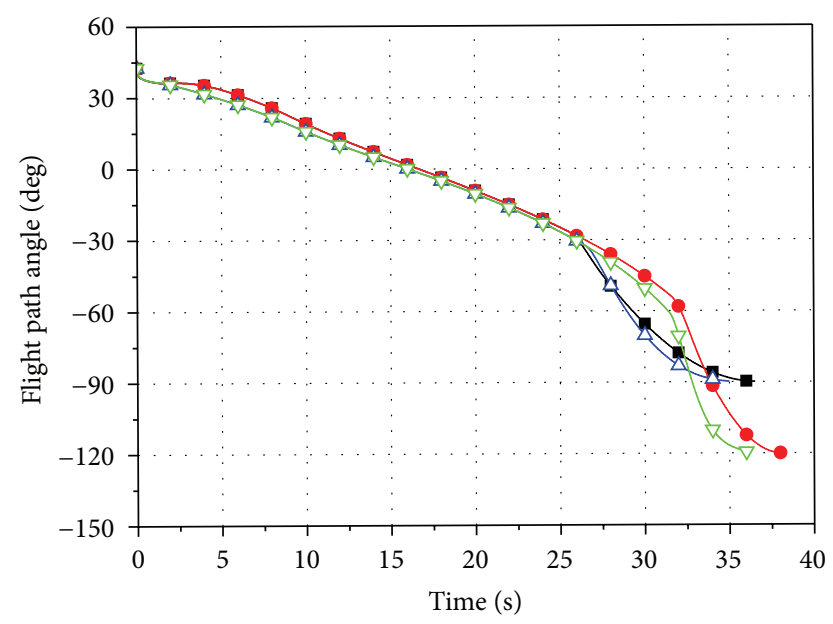

$\rightarrow$ TVBPN, $\theta_{f}=-90 \mathrm{deg} \quad-\triangle$ SGPNG, $\theta_{f}=-90 \mathrm{deg}$ $\rightarrow-$ TVBPN, $\theta_{f}=-120 \mathrm{deg} \quad \rightarrow-$ SGPNG, $\theta_{f}=-120 \mathrm{deg}$

(b) Flight path angle

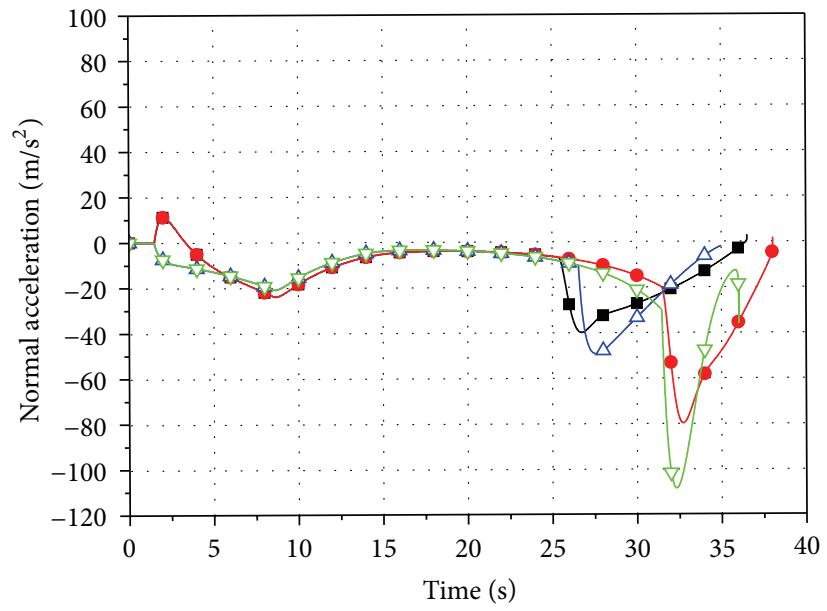

$\rightarrow-$ TVBPN, $\theta_{f}=-90 \mathrm{deg} \quad \triangle-\mathrm{SGPNG}, \theta_{f}=-90 \mathrm{deg}$ $\rightarrow$ TVBPN, $\theta_{f}=-120 \mathrm{deg} \quad \rightarrow$ SGPNG, $\theta_{f}=-120 \mathrm{deg}$

(d) Acceleration

FIGURE 6: Comparison results for realistic missile model.

TABLE 1: Simulation results between two guidance laws.

\begin{tabular}{lcc}
\hline Guidance laws & Impact angle error/deg & Miss distance/m \\
\hline TVBPN, -90 deg & 0.1048 & 0.0012 \\
TVBPN, $-120 \mathrm{deg}$ & 0.1199 & 0.0434 \\
BGPNG,-90 deg & 0.1389 & 0.0004 \\
BGPNG,-120 deg & 0.3374 & 0.0683 \\
\hline
\end{tabular}

the target and achieve terminal angle control with negligible error. The maximum acceleration of TVBPN is relatively smaller than that of SGPNG, as shown in Figure 6(d).

\section{Conclusion}

A time-varying biased proportional navigation guidance law is designed in this study for achieving terminal angles control without violating the filed-of-view limit. The trajectory is shaped using two time-varying biases, $b_{1}$ and $b_{2}$. The firststage bias aims to keep the seeker's lock-on during the engagement. The second-stage bias ensures the terminal impact angle constraint based on the requirement that the integral of bias is equal to the expected bias amount. The initial and final phases of the engagement are undertaken with $b_{1}$ whereas $b_{2}$ comes into play if the switching condition is satisfied. The missile-target relative range at the switching instant and the lateral acceleration requirement are also derived. The analytical calculation may be a good guideline for engineers to design in advance. Finally, simulations are carried out for kinematic and realistic missile models separately to verify the performance of the proposed guidance law. The proposed method is more practical in passive homing missiles since it does not need relative range information or time-to-go estimation. And the time-varying bias ensures that the lateral 
acceleration of the proposed scheme changes continuously during the engagement. Future work will consider the biased guidance law for interception of maneuvering targets with angle constraint.

\section{Conflict of Interests}

The authors declare that there is no conflict of interests regarding the publication of this paper.

\section{Acknowledgments}

This work is supported by the National Natural Science Foundation of China (Grant no. 61172182) and the Science Innovation Foundation of Beijing Institute of Technology (Grant no. 2015CX10002).

\section{References}

[1] L. Yan, J. Zhao, H. Shen, and Y. Li, "Three-dimensional united biased proportional navigation law for interception of maneuvering targets with angular constraint," Proceedings of the Institution of Mechanical Engineers, Part G: Journal of Aerospace Engineering, vol. 229, no. 6, pp. 1013-1024, 2015.

[2] R. K. Mehra and R. D. Ehrich, "Air-to-air missile guidance for strapdown seekers," in Proceedings of the 23rd IEEE Conference on Decision and Control, pp. 1109-1115, IEEE, Las Vegas, Nev, USA, December 1984.

[3] C. H. Lee, C. Hyun, J. G. Lee, J. Y. Choi, and S. Sung, "A hybrid guidance law for a strapdown seeker to maintain lockon conditions against high speed targets," Journal of Electrical Engineering and Technology, vol. 8, no. 1, pp. 190-196, 2013.

[4] C.-K. Ryoo, H. Cho, and M.-J. Tahk, "Optimal guidance laws with terminal impact angle constraint," Journal of Guidance, Control, and Dynamics, vol. 28, no. 4, pp. 724-732, 2005.

[5] H. Wang, D. Lin, Z. Cheng, and J. Wang, "Optimal guidance of extended trajectory shaping," Chinese Journal of Aeronautics, vol. 27, no. 5, pp. 1259-1272, 2014.

[6] C.-K. Ryoo, H. Cho, and M.-J. Tahk, "Time-to-go weighted optimal guidance with impact angle constraints," IEEE Transactions on Control Systems Technology, vol. 14, no. 3, pp. 483-492, 2006.

[7] Y. Zhang, M. Sun, and Z. Chen, "Finite-time convergent guidance law with impact angle constraint based on slidingmode control," Nonlinear Dynamics, vol. 70, no. 1, pp. 619-625, 2012.

[8] S. R. Kumar and D. Ghose, "Three dimensional impact angle constrained guidance law using sliding mode control," in Proceedings of the American Control Conference (ACC '14), pp. 2474-2479, IEEE, Portland, Ore, USA, June 2014.

[9] S. He, D. Lin, and J. Wang, "Continuous second-order sliding mode based impact angle guidance law," Aerospace Science and Technology, vol. 41, pp. 199-208, 2015.

[10] P. Zarchan, Tactical and Strategic Missile Guidance, AIAA, Reston, Va, USA, 6th edition, 2012.

[11] B. S. Kim, J. G. Lee, and H. S. Han, "Biased PNG law for impact with angular constraint," IEEE Transactions on Aerospace and Electronic Systems, vol. 34, no. 1, pp. 277-288, 1998.

[12] Y. Myung-Gon, "Relative circular navigation guidance for the impact angle control problem," IEEE Transactions on Aerospace and Electronic Systems, vol. 44, no. 4, pp. 1449-1463, 2008.
[13] A. Ratnoo and D. Ghose, "Impact angle constrained interception of stationary targets," Journal of Guidance, Control, and Dynamics, vol. 31, no. 6, pp. 1817-1822, 2008.

[14] K. S. Erer and O. Merttopçuoglu, "Indirect impact-anglecontrol against stationary targets using biased pure proportional navigation," Journal of Guidance, Control, and Dynamics, vol. 35, no. 2, pp. 700-703, 2012.

[15] T.-H. Kim, B.-G. Park, and M.-J. Tahk, "Bias-shaping method for biased proportional navigation with terminal-angle constraint," Journal of Guidance, Control, and Dynamics, vol. 36, no. 6, pp. 1810-1816, 2013.

[16] A. Ratnoo, "Analysis of two-stage proportional navigation with heading constraints," Journal of Guidance, Control, and Dynamics, vol. 39, no. 1, pp. 156-164, 2016.

[17] K. S. Erer, R. Tekin, and M. K. Ozgoren, "Look angle constrained impact angle control based on proportional navigation," in Proceedings of the AIAA Guidance, Navigation, and Control Conference, AIAA, Kissimmee, Fla, USA, January 2015.

[18] N. A. Shneydor, "Proportional navigation-missile guidance and pursuit-5," Missile Guidance \& Pursuit, vol. 5, no. 3, pp. 101-127, 1998.

[19] P. Kee, L. Dong, and C. Siong, "Near optimal midcourse guidance law for flight vehicle," in Proceedings of the 36th AIAA Aerospace Sciences Meeting and Exhibit, American Institute of Aeronautics and Astronautics, Reno, Nev, USA, 1998. 


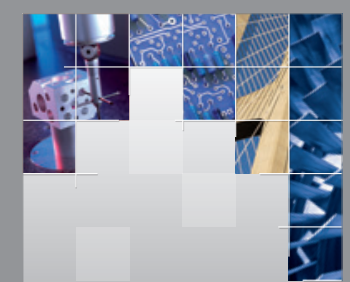

\section{Enfincering}
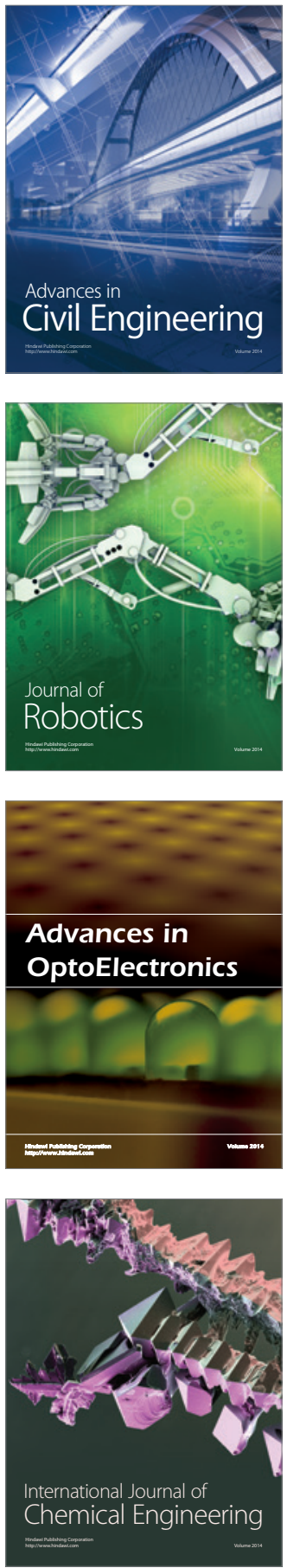

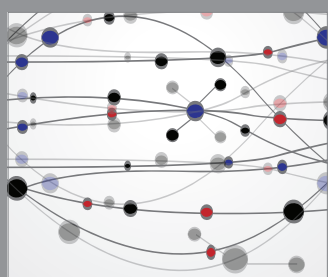

The Scientific World Journal

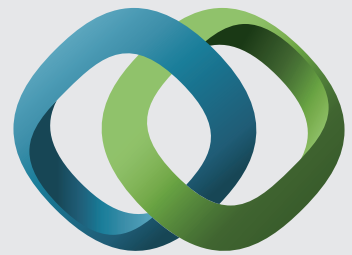

\section{Hindawi}

Submit your manuscripts at

http://www.hindawi.com
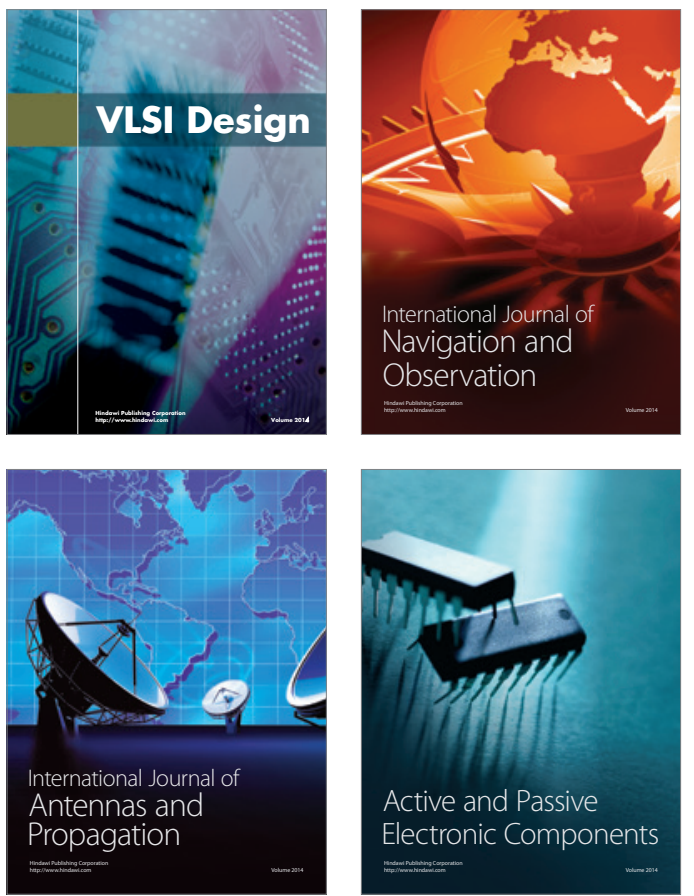
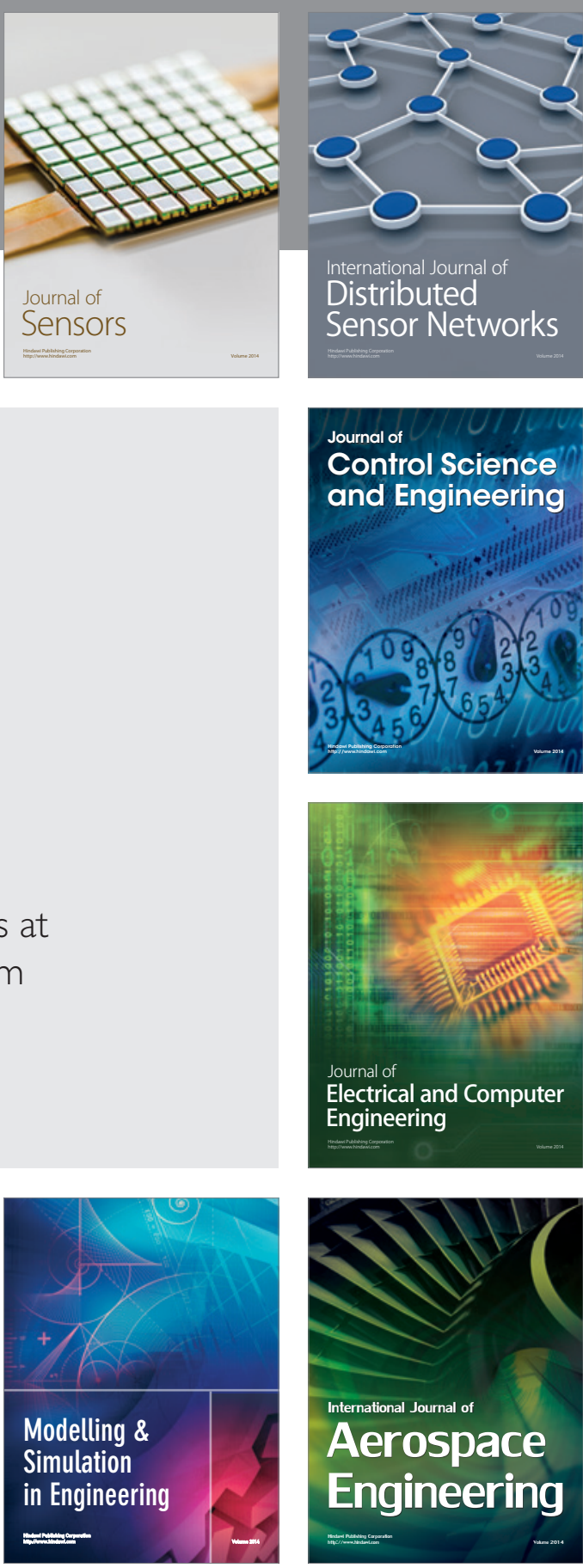

International Journal of

Distributed

Sensor Networks

Journal of

Control Science

and Engineering
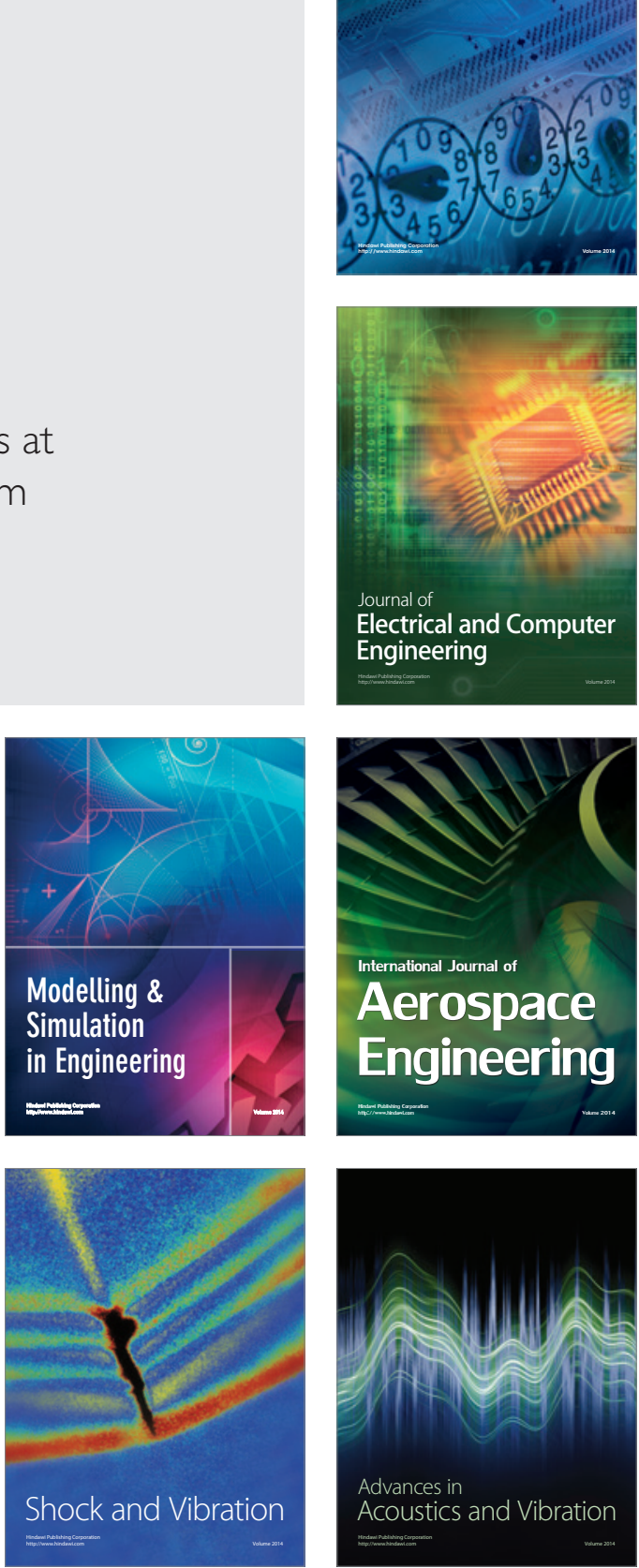